\title{
Comprehensive Information System on Mobile Devices via Bluetooth Application Server
}

\begin{abstract}
Abbas Ali Lotfi Neyestanak
Telecommunication Engineering \& IT Group, Iranian Research Institute for Electrical Engineering, ACECR, Tehran, Iran. Email: alotfi@iust.ac.ir

Received December $30^{\text {th }}, 2009$; revised March 20 $0^{\text {th }}, 2010$; accepted May $5^{\text {th }}, 2010$.

ABSTRACT

Advantage of cell phones and their rapid progress on facilities and applications, made a technical trend to develop software implementations for this kind of systems. On the other hand, Java package with some efficient tools and products made it quite desirable for producers in a wide range of applications. In this paper the Bluetooth technology has been studied and its defects and premiums and security threats are investigated and compared with other technologies. Finally a comprehensive information system is designed, simulated and implemented based on cell phone communications. This system actually utilizes the Bluetooth technology to send and receive variety of packets such as data, image, and sound between computer and cell phone. These transferred data are then processes at the either sides and the revealed data is presented to the user. Wide 100 meter coverage has been considered for this system utilizing advanced automatic traffic management routines.
\end{abstract}

Keywords: Information System, Bluetooth, JAVA, Mobile Phone

\section{Introduction}

Bluetooth is a short range wireless technology which provides communications capabilities to mobile phones, Personal Digital Assistants (PDAs), computers, record/ stereo systems and home appliances. Original idea of Bluetooth dates back to 1994 and was first proposed in Ericsson Company. At that time, Ericsson was attempting to develop a low cost radio communication between a mobile phone and a wireless handset. Technical process started in 1995 and it moved beyond the mobile phones and extended further to include any kinds of mobile equipment.

Bluetooth which provides a communication network between different small personal devices named after one of the Danish kings living in 940-981 AD. During his rule, King Harald who was a Viking, tried to peacefully unite Denmark, South Sweden and North Norway. This made him a famous king in history for his negotiation and communications skills. Therefore, Ericsson which also aimed to peacefully unite all different equipment found this name a best fit for this technology.

Ericsson knew that if only one company supports this standard, it cannot be successful and therefore, in 1998, Ericsson signed a contract with IBM, Intel, Nokia,
COM3, Toshiba and Microsoft and established Bluetooth special interest group (Bluetooth SIG).

The main objectives of this group were to supervise Bluetooth progress and to make it widely accepted. Although the main purpose of this technology was to have more freedom by having no cables between digital systems, soon it extended further and included other systems such as Wireless Local Area Network (WLAN) which are commonly used. However, some challenges arouse which correspond to common frequency of this technology and 802.11 standards which require being solved [1].

In July 1999, Bluetooth planning team published the first version of the specifications in 1500 pages. Soon, IEEE Personal Local Area (PAN) standard group who were willing to implement 802.15 standards chose Bluetooth documents as the reference draft and started to improve it. At first glance, it may seem pointless to standardize a technology the specifications of which already exist and has no diverse or incompatible deployments. However, in general, an open standard implemented by an impartial organization such as IEEE can facilitate and encourage use of a technology [2].

Although in 2002, IEEE approved the first PAN standard (802.15.1), Bluetooth consortium is willing to improve and extend it further. 3rd generation communica- 
tions networks are designed to allow multimedia transmission which consists of voice, data, image and any combinations of all was has different bit rate and quality of service.

In $802.11 \mathrm{~b}$ networks, data rate can be 11, 5.5, 2 and 1 $\mathrm{Mb} / \mathrm{s}$. For 1 and $2 \mathrm{Mb} / \mathrm{s}$, DBPSK and DQPSK modulations are used whereas for 5.5 and $11 \mathrm{Mb} / \mathrm{s}$ Complementary Code Keying (CCK) modulation is used. In $802.11 \mathrm{~g}$ networks which are the extensions of $802.11 \mathrm{~b}$, data rate has been increased to $54 \mathrm{Mb} / \mathrm{s}$ due to OFDM modulation [3].

In WLAN systems, in order to transmit and receive data, $22 \mathrm{MHz}$ bandwidth is required; therefore in standard bandwidth of $89 \mathrm{MHz}$, there are three $25 \mathrm{MHz}$ channels available $[4,5]$.

Several researchers have investigated the issue of error control code design for Bluetooth systems [6-8]. Most of them have come to the conclusion that error control codes implemented for this task are not powerful for fading channels [9].

Channel coding is required for wireless communications to protect data from the errors which may result from noise and interference. In the Bluetooth system, there are several channel coding schemes that are implemented. The purpose of the channel coding scheme on the data payload is to reduce the retransmission times which are due to channel errors $[10,11]$.

Bluetooth communication is always client-server based, i.e., one device (client) tries to make use of the services offered by another device (server).

Bluetooth LAN connections, which are fairly common today, are used for interconnecting small devices such as printers, PDA's, Wireless network for microphone, keyboard, mouse, measurement and test equipment, medical instruments and notebook computers simply for data sharing. This paper investigates the information system on mobile devices via Bluetooth application server providing enterprise intranet services to mobile devices.

In fact the objective of this paper was to develop a comprehensive, information system for on-site propaganda facilities. The proposed structure based on Bluetooth technology can be applied to different systems such as public information, educations, control, automation and advertisement.

Since Bluetooth-based systems have become widespread and are free of charge, this system is very convenient to use for short range applications.

The software of the proposed system is based on Java and is compatible to most of operational systems on cell phones and personal computers. In addition, the system security is significantly high.

\section{Bluetooth Layers}

Bluetooth structure consists of different layers, as shown in Figure 1. Two main layers of this technology are RF and baseband layers which are responsible for most tasks. Baseband layer is a part of Bluetooth physical layer. It manages physical channels and some links such as error correction, data aligning, and frequency hopping selection and Bluetooth security. Baseband layer is on top of radio layer in Bluetooth layer configuration. Baseband layer is on top of the radio layer in Bluetooth layer configuration. It is also called link controller. This layer communicates with its upper layer, link manager so frequently that they cannot exist without each other. Both LM and LC manage the link by making connections and controlling the power. Baseband layer also manages synchronization connection of received packets and other tasks such as searching Bluetooth system, collecting basic information from Bluetooth system to access and data transfer in this layer is performed in certain time slots, Time Division Duplex (TDD).

\subsection{Physical Channel}

Bluetooth operates at $2.4 \mathrm{GHz}$ frequency range. In U.S., Europe and most countries, bandwidth of $83.5 \mathrm{MHz}$ is used which can be divided to $79,1 \mathrm{MHz}$ channels. In France and Japan, smaller bandwidth is used which is divided to 23, $1 \mathrm{MHz}$ channels. As shown in Figure 2, frequency hopping can be performed by pseudo-random method which allows two or more Bluetooth system to be able to use the same radio channels within a piconet. In one piconet, there are one or more slaves and one main master. Frequency hopping steps for each piconet is unique and determined by address of Bluetooth master system.

\subsection{Bluetooth Radio Layer}

This layer is the lowest layer of Bluetooth protocol and

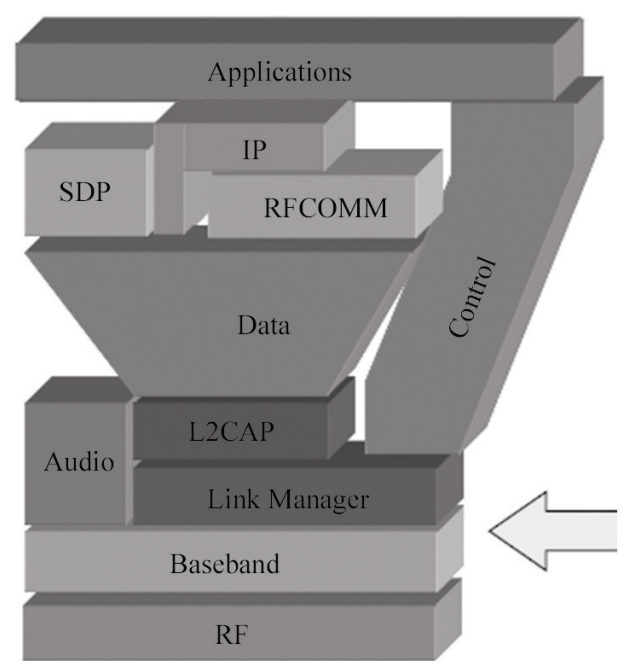

Figure 1. Bluetooth layers. 


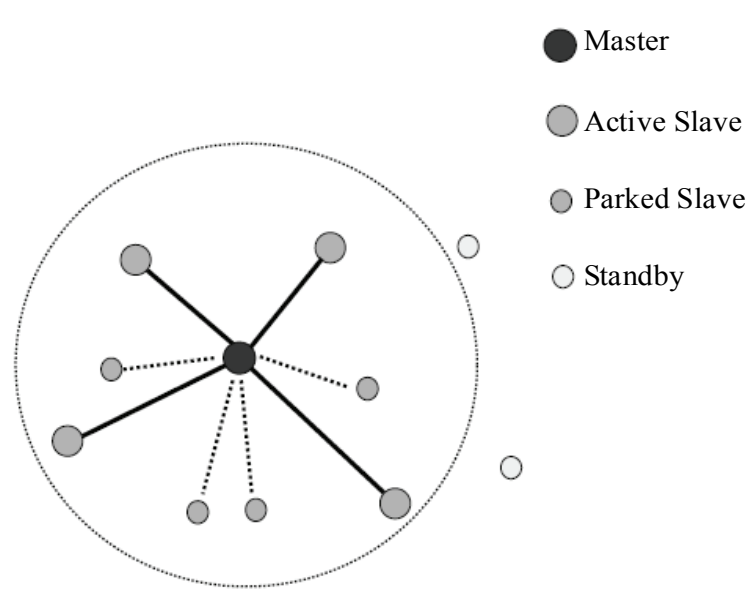

Figure 2. Piconet configuration.

defines transmitter and received properties. There are different block diagrams for designing Bluetooth RF layer and they show different parts such as input data to VCO, I/Q modulator and demodulator. Bluetooth system consists of RF unit, baseband control link unit and link management software. Figure 3 shows typical Bluetooth transmitter and receiver which includes RF and baseband units. Each of them has its own advantages and disadvantages. For instance, I/Q modulator and demodulator system can be implemented digitally and also it is able to cancel image frequency, however its output waveform is a little distorted.

On the other hand, in VCO modulator and demodulator system there is no distortion on the output waveform. However it is an analogue system and image frequency does exist.

\section{System Development}

A block diagram of this system is shown in Figure 4. The hardware of this system is tried to keep minimum and cheap. The physical operating range is adjustable to maximum of $230 \mathrm{~m}$ and transceiver antenna is adapted to the area.

The server continuously searches area to find any new user and in case of finding any, server would give it a unique ID and in some cases a copy of the application program.

Server recognizes the difference between laptops or PDAs and sends them the appropriate version of the software. Varieties of file formats are applicable in this system including audio, video, text, and...

All of the software forms and windows are designed based on multi language support and swing platform. This guarantees the form profile to be seen exactly the same in any OS and PC.

\subsection{Bluetooth System Connection Using JSR82 Standard}

JSR82 standard has first been presented by Motorola Company to be used in J2ME structure of cell phones. This API is able to connect to the following Bluetooth stacks: Microsoft Bluetooth stack (Windows), WIDCOMM Bluetooth Stack (Windows), Bluesoleil Bluetooth stack (Windows), BlueZ Bluetooth Stack (Linux), and Mac OS Bluetooth stack (OS x). It also uses Apache Derby database server which earns the system a series of advantages including platform independency, server base and embedded presentation, fair performance, and default in Java v. 6.

\subsection{System Traffic Management}

According to constrain of 7 simultaneous connections, software implements an advance system to manage the data transfer. Channel dedication procedure to a typical user is a multi thread execution which occurs as follows.

First of all new users are searched and determined by using a service named device inquiry service. This service limits itself to a certain maximum number of new clients and the rest of them will be left to the next immediate searching. This maximum number of recognized (searched) users $\left(\mathrm{N}_{\mathrm{Users}}\right)$ calculates by Equation (1).

$$
N_{\text {Useres }}=2 \times N_{\text {Channels }}-N_{O C}
$$

where $N_{\text {Channels }}$ is 7 in general cases and $N_{O C}$ is the number of occupied channels which are already used to send data to another user(s). This equation just uses when the number of client is more than $N_{\text {Channels. }}$. When inquiry finishes, the vacant channels dedicates to new users in queue and this process repeats until the last one in the line. In the other hand, the described process has a timeout parameter which limits the suspension time of data transferring. After this timeout, the search will start again and users in previous queue have priority.

\subsection{System Report Management}

All system transactions including successful submissions, unsuccessful submissions, and registered users are logged in the main database server and could be retrieved or reported. A scene of this part of the program has presented in Figure 5.

Furthermore, this application has a fully automatic error recording procedures which saves the error messages happen during system operation. These data could then be sending to the software developer for further obviation, improvements, and optimization.

\subsection{Manual and Timed Submission Modules}

Using one of these modules; as shown in Figure 6, user 


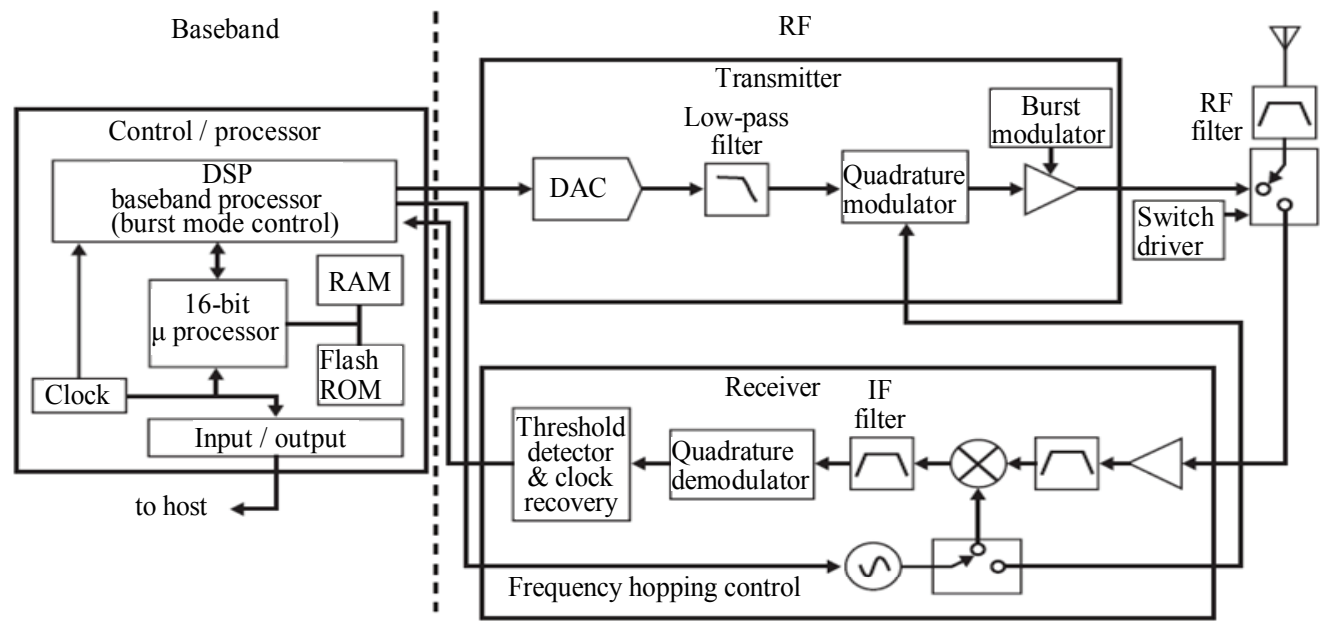

Figure 3. Block diagram of the RF and baseband sections.

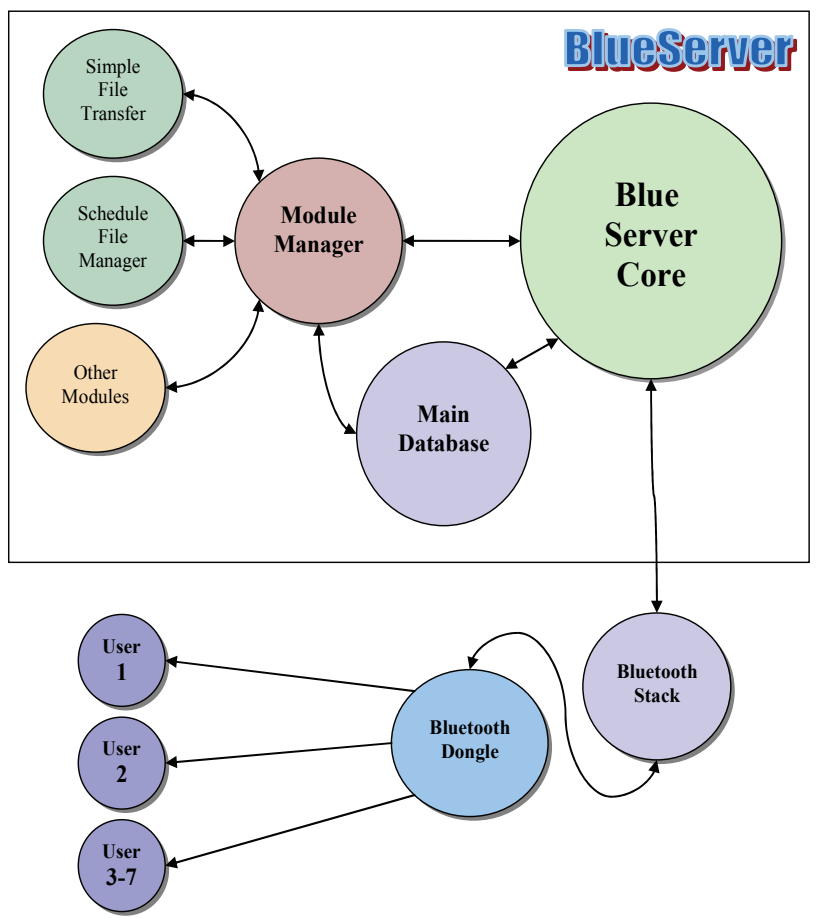

Figure 4. System block diagram.

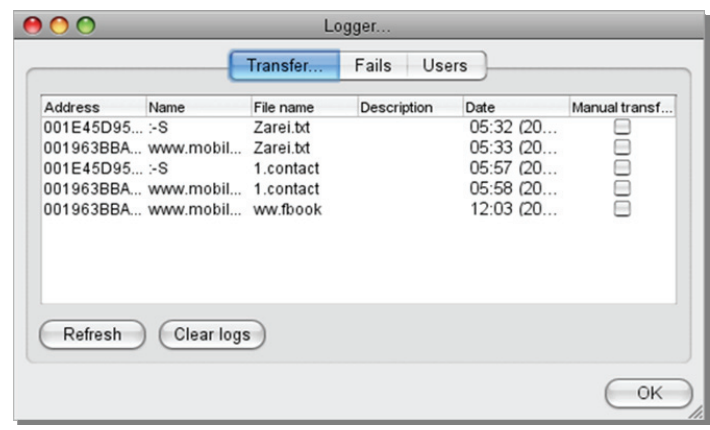

Figure 5. System report management and logging. could easily select and transmit any desired files and submit it automatically during a specific time period. This software is also able to connect to Contact Frame Creator and also Magic fBook Creator to create any desired text, image, map, contact sheet, SMS, news, and...

\subsection{Adjust Setting}

Program's main view is shown in Figure 7(a). By choosing the menu item Files $\rightarrow$ Settings the window of Figure 7(b) will appear where user could adjust the searching and submission time intervals, activate or deactivate software alarm system which uses balloons in system try region of OS, and...

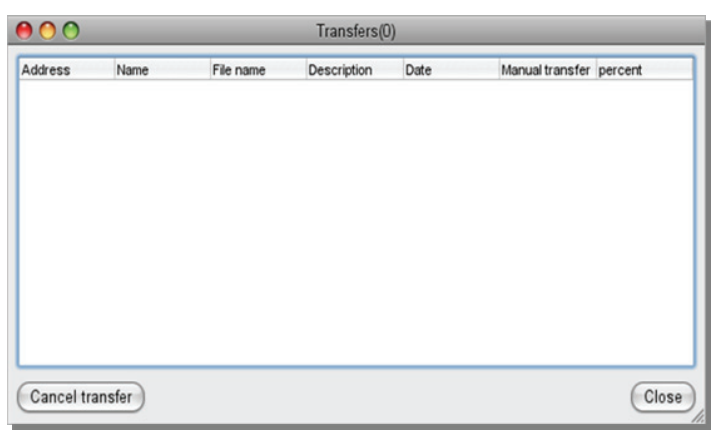

(a)

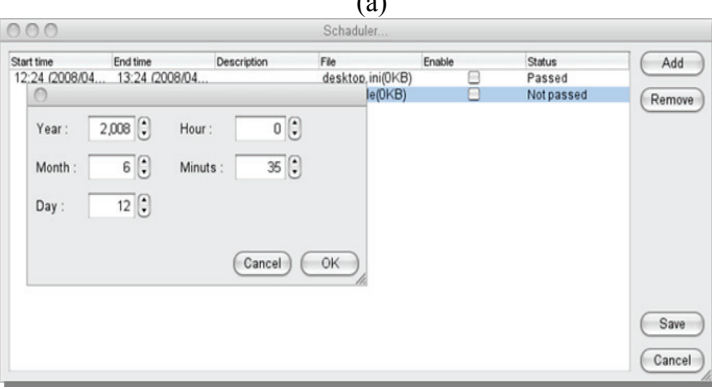

(b)

Figure 6. (a) Manual; (b) Timed submission module. 


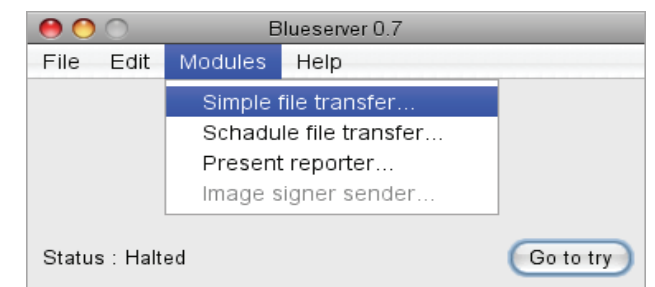

(a)

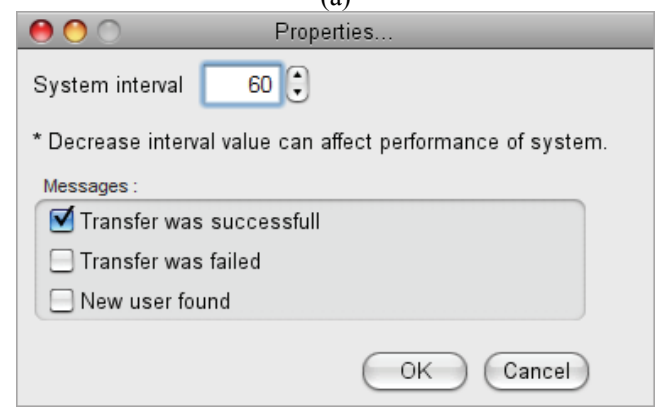

(b)

Figure 7. (a) Bluetooth server program; (b) Its setting window.

\section{Conclusions}

The Bluetooth technology has been studied and investigated to realize its defects and threats and then a comprehensive information system is designed, simulated and implemented based on cell phone communications. Hereby Bluetooth technology is utilized to send and receive variety of packets and files between computer and cell phone. JAVA technology and it's relevant like J2EE and JAVA application are used here which helps system developers to produce cell phone-based applications and data transferring on any platform and OS. Firstly 100 meter coverage considered for this system which is extendable to $230 \mathrm{~m}$ and more. An advanced automatic traffic management routines has also been defined and used.

\section{REFERENCES}

[1] http://www.bluetooth.com/

[2] Specification of the Bluetooth System, Version 1.1, Bluetooth SIG, 22 February 2001.

[3] Agilent Technologies, "Wireless Networking Design and Verification," Wireless Networking Design Seminar, 17 October 2001 .

[4] IEEE 802.11, "the Working Group Setting the Standards for Wireless Lens.” http:// grouper.ieee.org/groups/802/11

[5] B. Razavi, "Monolithic Phase-Locked Loops and Clock Recovery Circuits," IEEE Press, New Jersey, 2004.

[6] I. Howitt, "Bluetooth Performance in the Presence of 802.11b WLAN," IEEE Transactions on Vehicular Technology, Vol. 51, No. 6, November 2002, pp. 1640-1651.

[7] "To Learn More about Practical Applications of Wireless Network Simulation." http:// www. iwtwireless.com

[8] S. Galli, D. Famolari and T. Kodama, "Bluetooth: Channel Coding Considerations," IEEE Vehicular Technology Conference, Vol. 5, 17-19 May 2004, pp. 2605-2609.

[9] A. Conti, D. Dardari, G. Paolini and O. Andrisano, "Bluetooth and IEE 802.11b Coexistence: Analytical Performance Evaluation in Fading Channels," IEEE Journal on Selected Areas in Communications, Vol. 21, No. 2, February 2003, pp. 259-269.

[10] N. Golmie, R. E. van Dck and A. Soltanian, "Interference of Bluetooth and IEEE 802.11: Simulation Modeling and Performance Evaluation," Proceedings ACM International Workshop on Modeling, Analysis, and Simulation of Wireless and Mobile Systems, Rome, July 2001, pp. 11-18.

[11] L. Ozarow, S. Shamai and A. D. Wyner, "Information Theoretic Considerations for Cellar Mobile Radio," IEEE Transactions on Vehicular Technology, Vol. 43, No. 2, May 1994, pp. 359-378. 